\title{
Stability of neutral-type descriptor systems with multiple time-varying delays
}

\author{
Yuxia Zhao ${ }^{*}$ and Yuechao Ma
}

* Correspondence: zhaoyuxiafei@126.com College of Science, Yanshan University, Qinhuangdao Hebei 066004, P. R. China

\begin{abstract}
This article deals with the problem of stability of descriptor neutral systems with multiple delays. Using Lyapunov functional and free-weighting matrix method, a delay-dependent stability criterion is obtained and formulated in the form of linear matrix inequalities, which can easily be checked by utilizing Matlab linear matrix inequality toolbox. Finally, a numerical example is presented to illustrate the effectiveness of the method.
\end{abstract}

Keywords: neutral-type descriptor systems, asymptotical stability, free-weighting matrix, linear matrix inequality

\section{Introduction}

Since the time delay is frequently viewed as a source of instability and encountered in various engineering systems such as chemical processes, long transmission lines in pneumatic systems, networked control systems, etc., the study of delay systems has received much attention and various topics have been discussed over the past years. Commonly, the existing results can be classified into two types: delay-independent conditions and delay-dependent conditions. In general, the delay-dependent case is more conservative than delay-independent case.

A neutral system with time-delays which contains delays both in its state and in its derivatives of state is encountered in many dynamic systems and their presences must be taken into account in real dynamic process such as circuit systems, population dynamics, automatic control, and heat exchangers, etc. Due to its profound and practical background, much attention has been focused on the problems of stability analysis for neutral time-delay system from mathematics and control communities [1-7]. Using Lyapunov method, Park [1] presented new sufficient conditions for the stability of the systems in terms of linear matrix inequality (LMI) which can be easily solved by various convex optimization algorithms. Some delay-independent stability criteria were given in terms of the characteristic equation of system, involving the measures, eigenvalues, spectral radius, and spectral norms of the corresponding matrices [3]. Although the conditions are easy to check, they require the matrix measure to be Hurwitz matrix. The problem of delay-dependent stability criteria for a class of constant timedelay neutral systems with time-varying structured uncertainties was investigated [4]. Han [5] obtained delay-dependent stability conditions for uncertain neutral time-varying system by model transformation method, due to cross terms of model

(c) 2012 Zhao and Ma; licensee Springer. This is an Open Access article distributed under the terms of the Creative Commons Attribution License (http://creativecommons.org/licenses/by/2.0), which permits unrestricted use, distribution, and reproduction in any medium, provided the original work is properly cited. 
transformation, results are less conservative. Zhao [6] dealt with the problem of delaydependent robust stability for delay neutral type control system with time-varying structured uncertainties and time-varying delay. Some new delay and its derivativedependent criteria were derived. He [7] concerned the problem of the delay-dependent robust stability of neutral systems with mixed delays and time-varying structured uncertainties. A new method based on linear matrix inequalities was presented that makes it easy to calculate both the upper stability bounds on the delays and the free weighting matrices. Since the criteria take the sizes of the neutral- and discrete-delays into account, it is less conservative than previous methods.

Recently, Li [8] studied the stability of the neutral-type descriptor system with mixed delays, and derived some stability criteria, but the criteria are all delay independent which do not include the information on delay, therefore have a some conservative in. However, the descriptor delay neutral system stability and control have not yet fully investigated, and their stability conditions are not given a strict linear matrix inequalities, it is difficult to achieve through the LMI toolbox in Matlab. Particularly delaydependent sufficient conditions are few even non-existing in the published works.

In this article, the problem of stability of neutral type descriptor systems with timevarying delays is researched. Using free-weight matrix method in combination with Lyapunov-Krasovskii functional method is used to obtain the LMI-based delay-dependent sufficient conditions for stability. And we consider parameter uncertainties both in its state and in the derivatives of its state. Examples are given to illustrate the effectiveness of the condition.

Notations: The notation in this article is quite standard. $\mathrm{R}^{n}$ and $\mathrm{R}^{n \times m}$ denote, respectively the $n$-dimensional Euclidean space and the set of all $n \times m$ real matrices. The superscript $X^{\mathrm{T}}$ and $X^{-1}$ denote, respectively, the transpose and the inverse of any square matrix $X . I$ is the identity matrix of appropriate dimension. $\|\cdot\|$ will refer to the Euclidean vector norm. The symbol "always denotes the symmetric block in one symmetric matrix.

\section{System description}

Consider the following uncertain neutral type descriptor time-delay systems:

$$
\left\{\begin{array}{c}
E \dot{x}(t)-\sum_{i=1}^{m}\left(D_{i}+\Delta D_{i}\right) \dot{x}\left(t-h_{i}(t)\right)=\left(A_{0}+\Delta A_{0}\right) x(t)+\sum_{i=1}^{m}\left(A_{i}+\Delta A_{i}\right) x\left(t-d_{i}(t)\right) \\
x(t)=\varphi(t), \quad t \in[-\max \{h, d\}, 0]
\end{array}\right.
$$

where $x(t) \in \mathrm{R}^{n}$ is the state, $\phi(t)$ is a continuous vector-valued initial function, $\begin{aligned} 0 & <h_{1}(t)<h_{2}(t)<\cdots<h_{m}(t) \leq h, 0=d_{0}(t)<d_{1}(t)<\cdots<d_{m}(t) \leq d, 0<, A_{0}, A_{i}, D_{i} \\ \dot{h}_{t}(t) & <\tau_{i}(t)<\tau_{i} \leq \tau \leq 1,0<\dot{d}_{i}(t)<\mu_{i} \leq \mu \leq 1\end{aligned}$

$\in \mathrm{R}^{n}, A_{i}, D_{i}$ are known constant matrices with appropriate dimensions. Where $\Delta A_{i}$, $\Delta D_{i}$ are the constant matrices which denote time-varying parameter uncertainties and are assumed to belong to certain bounded compact sets. The parameter uncertainties are assumed to be of the following form:

$$
\left[\Delta A_{0}(t) \quad \Delta A_{i}(t) \quad \Delta D_{i}(t)\right]=H F(t)\left[\begin{array}{lll}
E_{0} & E_{i 1} & E_{i 2}
\end{array}\right]
$$

where $H, E_{i k}(k=1,2)$ are known real constant matrices with appropriate dimensions, and $F(t)$ is the uncertain matrix satisfying $F^{\mathrm{T}}(t) F(t) \leq I, \forall t$, I is unit matrix with appropriate dimensions.

Remark 1. When $E=I$, the system (1) reduces to the traditional uncertain neutral system with time-varying delays. 
Remark 2. Li [8] considered the stability of neutral type descriptor systems with constant time-delay, and the system did not include parameter uncertainty in the derivative of its state. So the system (1) is more widely in our article.

Lemma 1 (Schur-complement) For any matrix $S=\left[\begin{array}{ll}S_{11} & S_{12} \\ S_{12}^{T} & S_{22}\end{array}\right]$, with $S_{11}=S_{11}^{\mathrm{T}}, S_{12}=S_{12}^{\mathrm{T}}$, then the following conditions are equivalent:

$$
(1) S<0, \quad(2) S_{11}<0, \quad S_{22}-S_{12}^{\mathrm{T}} S_{11}^{-1} S_{12}<0, \quad(3) S_{22}<0, \quad S_{11}-S_{12} S_{22}^{-1} S_{12}^{\mathrm{T}}<0
$$

Lemma 2 [9] If there is symmetric matrix $X,\left[\begin{array}{cc}P_{1}+X & Q_{1} \\ Q_{1}^{T} & R_{1}\end{array}\right]>0,\left[\begin{array}{cc}P_{2}+X & Q_{2} \\ Q_{2}^{T} & R_{2}\end{array}\right]>0$ if and only if

$$
\left[\begin{array}{ccc}
P_{1}+P_{2} & Q_{1} & Q_{2} \\
Q_{1}^{\mathrm{T}} & R_{1} & 0 \\
Q_{2}^{\mathrm{T}} & 0 & R_{2}
\end{array}\right]>0
$$

Lemma 3 [10] Given matrices $Q=Q^{\mathrm{T}}, H, E$ with appropriate dimensions, we have

$$
Q+H F E+E^{\mathrm{T}} F^{\mathrm{T}} H^{\mathrm{T}}<0,
$$

for all $F(t)$ satisfying $F^{\mathrm{T}} F \leq I$ if and only if there exists a constant $\varepsilon>0$, such that

$$
Q+\varepsilon H H^{\mathrm{T}}+\varepsilon^{-1} E^{\mathrm{T}} E<0 .
$$

\section{Main results}

Theorem 1 The nominal system of the system (1) is asymptotically stable, if there exist nonsingular symmetric matrix $P$, and positive-definite symmetric matrices $Q_{i} S_{i} R_{i}$ and any appropriate dimensional matrices $N_{i 0}, N_{i j}, M_{i j}(i, j=1,2, \ldots, m)$, such that the following LMI holds:

$$
\begin{aligned}
& E^{\mathrm{T}} P=P^{\mathrm{T}} E \geq 0 \\
& {\left[\begin{array}{cccc}
\Omega & \Gamma^{\mathrm{T}} \bar{S} & \Gamma^{\mathrm{T}} \bar{R} & -\bar{N} \\
* & -S & 0 & 0 \\
* & * & -R & 0 \\
* & * & * & -R
\end{array}\right]<0}
\end{aligned}
$$

where

$$
\begin{aligned}
& \Omega=\left[\begin{array}{cccccc}
\Omega_{00} & \cdots & \Omega_{0 m} & \Omega_{0 m+1} & \cdots & \Omega_{02 m+1} \\
\vdots & \ddots & \vdots & \vdots & \ddots & \vdots \\
\cdot & \cdots & \Omega_{m m} & \Omega_{m m+1} & \cdots & \Omega_{m 2 m+1} \\
\cdot & \cdots & * & \Omega_{m+1 m+1} & \cdots & \Omega_{m+12 m+1} \\
\vdots & \ddots & \vdots & \vdots & \ddots & \vdots \\
* & \cdots & * & * & \cdots & \Omega_{2 m+12 m+1}
\end{array}\right], \bar{N}_{i}=\left[\begin{array}{c}
N_{i 0} \\
\vdots \\
N_{i m} \\
M_{i 1} \\
\vdots \\
M_{i m}
\end{array}\right]
\end{aligned}
$$

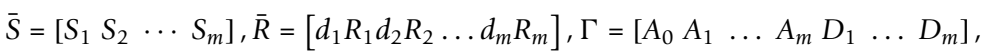

$$
\begin{aligned}
& \bar{N}=\left[d_{1} \bar{N}_{1} d_{2} \bar{N}_{2} \ldots d_{m} \bar{N}_{m}\right], S=\operatorname{diag}\left\{S_{1}, S_{2}, \ldots, S_{m}\right\}, R=\operatorname{diag}\left\{d_{1} R_{1}, d_{2} R_{2}, \ldots, d_{m} R_{m}\right\}, \\
& \Omega_{00}=P^{\mathrm{T}} A_{0}+A_{0}^{\mathrm{T}} P+\sum_{i=0}^{m}\left[N_{i 0} E+E^{\mathrm{T}} N_{i 0}^{\mathrm{T}}\right]+\sum_{i=1}^{m} Q_{i}, \Omega_{0 k}=P A_{k}-N_{k 0} E+\sum_{i=0}^{m} E^{\mathrm{T}} N_{i k^{\prime}}^{\mathrm{T}} \\
& \Omega_{0 m+k}=\mathrm{PD}_{k}+\sum_{i=0}^{m} E^{\mathrm{T}} M_{i k^{\prime}}^{\mathrm{T}} \Omega_{k k}=-\left(1-\mu_{i}\right) Q_{k}-\left(N_{k k} E+E^{\mathrm{T}} N_{k k}^{\mathrm{T}}\right), \quad k=1,2, \ldots, m, \\
& \Omega_{l m+k}=-E^{\mathrm{T}} M_{l k^{\prime}}^{\mathrm{T}} l, k=1,2, \ldots, m, \Omega_{l k}=-N_{k l} E-E^{\mathrm{T}} N_{l k^{\prime}}^{\mathrm{T}} \quad l=1,2, \ldots, m, \quad l<k \leq m, \\
& \Omega_{m+k m+k}=-\left(1-\tau_{i}\right) E^{\mathrm{T}} S_{k} E, \quad k=1,2, \ldots, m,
\end{aligned}
$$


Proof: Constructing a Lyapunov-Krasovskii functional as follows:

$$
V(x, t)=V_{1}+V_{2}+V_{3}+V_{4}
$$

in which

$$
\begin{gathered}
V_{1}=x^{\mathrm{T}}(t) E^{\mathrm{T}} P x(t), V_{2}=\sum_{i=0}^{m} \int_{t-d_{i}(t)}^{t} x^{\mathrm{T}}(s) Q_{i} x(s) d s, \\
V_{3}=\sum_{i=0}^{m} \int_{t-h_{i}(t)}^{t} \dot{x}^{\mathrm{T}}(s) E^{\mathrm{T}} S_{i} E \dot{x}(s) d s, V_{4}=\sum_{i=0}^{m} \int_{-d_{i}(t)}^{0} \int_{t+\theta}^{t} \dot{x}^{\mathrm{T}}(s) E^{\mathrm{T}} R_{i} E \dot{x}(s) d s d \theta,
\end{gathered}
$$

The time derivative of $V(x, t)$ along the trajectory of system (1) is given by

$$
\begin{gathered}
\dot{V}=\dot{V}_{1}+\dot{V}_{2}+\dot{V}_{3}+\dot{V}_{4} \\
\dot{V}_{1}=2 x^{\mathrm{T}}(t) P E \dot{x}(t)=2 x^{\mathrm{T}}(t) P\left(A_{0} x(t)+\sum_{i=0}^{m} A_{i} x\left(t-d_{i}(t)\right)+\sum_{i=0}^{m} D_{i} \dot{x}\left(t-h_{i}(t)\right)\right) \\
\dot{V}_{2}=\sum_{i=1}^{m} x^{\mathrm{T}}(t) Q_{i} x(t)-\sum_{i=1}^{m}\left(1-\mu_{i}\right) x^{\mathrm{T}}\left(t-d_{i}(t)\right) Q_{i} x\left(t-d_{i}(t)\right), \\
\dot{V}_{3}=\sum_{i=1}^{m} \dot{x}^{\mathrm{T}}(t) E^{\mathrm{T}} S_{i} E \dot{x}(t)-\sum_{i=1}^{m}\left(1-\tau_{i}\right) \dot{x}^{\mathrm{T}}\left(t-h_{i}(t)\right) E^{\mathrm{T}} S_{i} E \dot{x}\left(t-h_{i}(t)\right) \\
\dot{V}_{4}=\sum_{i=1}^{m} d_{i} \dot{x}^{\mathrm{T}}(t) E^{\mathrm{T}} R_{i} E \dot{x}(t)-\sum_{i=1}^{m} \int_{t-d_{i}(t)}^{t} \dot{x}^{\mathrm{T}}(s) E^{\mathrm{T}} R_{i} E \dot{x}(s) d s
\end{gathered}
$$

According to Newton-Leibniz formula, apparently for any appropriate dimensional matrices $\mathrm{N}_{i 0}, \mathrm{~N}_{i j}, M_{i j}(i, j=1,2, \ldots, m)$, then

$$
2 \sum_{i=0}^{m}\left[x^{\mathrm{T}}(t) N_{i 0}+\sum_{j=1}^{m} x^{\mathrm{T}}\left(t-d_{i}(t)\right) N_{i j}+\sum_{j=1}^{m} \dot{x}^{\mathrm{T}}\left(t-h_{j}(t)\right) \mathcal{M}_{i j}\right]\left[E x(t)-E x\left(t-d_{i}(t)\right)-\int_{t-d_{i}(t)}^{t} E \dot{x}(s) d s\right]=0,
$$

As for any appropriate dimensional matrix $X_{i} \geq 0,(i=1,2, \ldots, m)$, then

$$
\sum_{i=0}^{m}\left[\xi_{1}^{\mathrm{T}}(t) X_{i} \xi_{1}(t)-\int_{t-d_{i}(t)}^{t} \xi_{1}^{\mathrm{T}}(t) X_{i} \xi_{1}(t) d s\right]=0,
$$

where

$$
\begin{gathered}
X_{i}=\left[\begin{array}{cccccc}
X_{i 00} & \cdots & X_{i 0 m} & X_{i 0 m+1} & \cdots & X_{i 02 m+1} \\
\vdots & \ddots & \vdots & \vdots & \ddots & \vdots \\
\cdot & \cdots & X_{i m m} & X_{m m+1} & \cdots & X_{i m 2 m+1} \\
\cdot & \cdots & * & X_{i m+1 m+1} & \cdots & X_{i m+12 m+1} \\
\vdots & \ddots & \vdots & \vdots & \ddots & \vdots \\
* & \cdots & * & * & \cdots & X_{i 2 m+12 m+1}
\end{array}\right], \\
\xi_{1}^{\mathrm{T}}(t)=\left[x^{\mathrm{T}}(t) x^{\mathrm{T}}\left(t-d_{1}(t)\right) \ldots x^{\mathrm{T}}\left(t-d_{m}(t)\right) \dot{x}^{\mathrm{T}}\left(t-h_{m}(t)\right) \ldots \dot{x}^{\mathrm{T}}\left(t-h_{m}(t)\right)\right]
\end{gathered}
$$


from (6),(7) and (5), lead to

$$
\dot{V}=\xi_{1}^{\mathrm{T}}(t)\left(\Omega+\sum_{i=0}^{m} d_{i} X_{i}+\sum_{i=0}^{m} \Gamma^{\mathrm{T}}\left(S_{i}+d_{i} R_{i}\right) \Gamma\right) \xi_{1}(t)-\int_{t-d_{i}(t)}^{t} \xi_{2}^{\mathrm{T}}(t, s) \Psi_{i} \xi_{2}(t, s) d s
$$

in which

$$
\xi_{2}^{\mathrm{T}}(t, s)=\left[\xi_{1}^{\mathrm{T}}(t)(E \dot{x}(s))^{\mathrm{T}}\right], \quad \Psi_{i}=\left[\begin{array}{cc}
d_{i} X_{i} & d_{i} N_{i} \\
* & d_{i} R_{i}
\end{array}\right]
$$

If

$$
\left(\Omega+\sum_{i=0}^{m} d_{i} X_{i}+\sum_{i=0}^{m} \Gamma^{\mathrm{T}}\left(S_{i}+d_{i} R_{i}\right) \Gamma\right)<0, \quad \Psi_{i} \geq 0
$$

According to Lyapunov-Krasovskii stability theorem, the system (1) is asymptotically stable. According to Schur-complement,

$$
\begin{aligned}
& \left(\Omega+\sum_{i=0}^{m} d_{i} X_{i}+\sum_{i=0}^{m} \Gamma^{\mathrm{T}}\left(S_{i}+h_{i} R_{i}\right) \Gamma\right)<0 \\
& \Leftrightarrow\left[\begin{array}{ccc}
\Omega+\sum_{i=0}^{m} d_{i} X_{i} \Gamma^{\mathrm{T}} \bar{S} \Gamma^{\mathrm{T}} \bar{R} \\
* & -S & 0 \\
* & * & -R
\end{array}\right]<0, \\
& \Leftrightarrow\left[\begin{array}{ccc}
-\Omega-\sum_{i=0}^{m} d_{i} X_{i}-\Gamma^{\mathrm{T}} \bar{S}-\Gamma^{\mathrm{T}} \bar{R} \\
* & S & 0 \\
* & * & R
\end{array}\right]>0 \\
& \Psi_{i} \geq 0 \Leftrightarrow\left[\begin{array}{cc}
d_{i} X_{i} & d_{i} N_{i} \\
* & d_{i} R_{i}
\end{array}\right]>0
\end{aligned}
$$

According to Lemma 2, from (10) and (11), if and only if

$$
\left[\begin{array}{cccc}
-\Omega-\sum_{i=0}^{m} d_{i} X_{i} & -\Gamma^{\mathrm{T}} \bar{S} & \Gamma^{\mathrm{T}} \bar{R} & -\bar{N} \\
* & S & 0 & 0 \\
* & * & R & 0 \\
* & * & * & R
\end{array}\right]>0 \Leftrightarrow\left[\begin{array}{cccc}
\Omega & \Gamma^{\mathrm{T}} \bar{S} & \Gamma^{\mathrm{T}} \bar{R} & -\bar{N} \\
* & -S & 0 & 0 \\
* & * & -R & 0 \\
* & * & * & -R
\end{array}\right]<0
$$

Then, we can get the theorem easily.

According to Theorem 1 and Lemma 3, it can be generalized to its structure uncertain neutral generalized time-delay systems, we have the following theorem:

Theorem 2 The system (1) is robustly asymptotically stable, if there exists constant $\varepsilon_{1}$ $>0$, nonsingular symmetric matrix $P$, positive-definite symmetric matrices $Q_{i}, S_{i}, R_{i}$ and any appropriate dimensional matrices $N_{i 0}, N_{i j}, M_{i j}(i, j=1,2, \ldots, m)$, such that the following LMI holds:

$$
E^{\mathrm{T}} P=P^{\mathrm{T}} E \geq 0
$$




$$
\left[\begin{array}{cccccc}
\Omega & \Gamma^{\mathrm{T}} \bar{S} & \Gamma^{\mathrm{T}} \bar{R} & \varepsilon \Theta_{1} & \Theta_{2}^{\mathrm{T}} & -\bar{N} \\
* & -S & 0 & 0 & 0 & 0 \\
* & * & -R & 0 & 0 & 0 \\
* & * & * & -\varepsilon I & 0 & 0 \\
* & * & * & * & -\varepsilon I & 0 \\
* & * & * & * & * & -R
\end{array}\right]<0
$$

in which

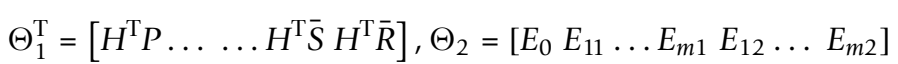

Proof: Replacing $A_{i}, D_{i}$ in (3b) with $A_{i}+\Delta A_{i}, D_{i}+\Delta D_{i}$ respectively, we find that (2) for (1) is equivalent to the following condition:

$$
\left[\begin{array}{cccc}
\Omega & \Gamma^{\mathrm{T}} \bar{S} & \Gamma^{\mathrm{T}} \bar{R} & -\bar{N} \\
* & -S & 0 & 0 \\
* & * & -R & 0 \\
* & * & * & -R
\end{array}\right]+\Theta_{1} F(t) \Theta_{2}+\Theta_{2}^{\mathrm{T}} F^{\mathrm{T}}(t) \Theta_{1}^{\mathrm{T}}<0
$$

By Lemma 3, a sufficient condition guaranteeing (13) for (1) is that there exists a positive number $\varepsilon>0$ such that

$$
\left[\begin{array}{cccc}
\Omega & \Gamma^{\mathrm{T}} \bar{S} & \Gamma^{\mathrm{T}} \bar{R} & -\bar{N} \\
* & -S & 0 & 0 \\
* & * & -R & 0 \\
* & * & * & -R
\end{array}\right]+\varepsilon \Theta_{1} \Theta_{1}^{\mathrm{T}}+\varepsilon^{-1} \Theta_{2}^{\mathrm{T}} \Theta_{2}<0
$$

Applying the Schur complement shows that (14) is equivalent to (12b). The proof is completed.

Remark 3. In the proof of the theorem, it is worth noting that the method taking the relationship between $E x(t)$ and $E x\left(t-d_{i}(t)\right)-\int_{t-d_{i}(t)}^{t} E \dot{x}(s) d s$ into account is suitable or deriving LMI conditions of the stability.

\section{Numerical examples}

Consider the system (1) described by

$$
\begin{gathered}
E=\left[\begin{array}{ll}
1 & 0 \\
0 & 0
\end{array}\right], \quad A_{0}=\left[\begin{array}{ll}
1 & 0.3 \\
1 & -2
\end{array}\right], \quad A_{1}=\left[\begin{array}{cc}
2 & 1 \\
-0.3 & 0.5
\end{array}\right], \quad A_{2}=\left[\begin{array}{cc}
0.5 & 0 \\
0 & -0.2
\end{array}\right], \quad D_{1}=\left[\begin{array}{cc}
0.1 & 0.3 \\
0 & 0.2
\end{array}\right], \quad D_{2}=\left[\begin{array}{cc}
0.2 & 0 \\
0.3 & 0.1
\end{array}\right], \\
H=\left[\begin{array}{cc}
0.1 & 0.5 \\
0.2 & -0.1
\end{array}\right], \quad E_{0}=\left[\begin{array}{cc}
-0.2 & 0.1 \\
0.1 & 0.3
\end{array}\right], \quad E_{1}=\left[\begin{array}{cc}
0.1 & 0 \\
0 & 0.3
\end{array}\right], \quad E_{2}=\left[\begin{array}{cc}
0.1 & 0.3 \\
-0.15 & 0.2
\end{array}\right], E_{3}=\left[\begin{array}{cc}
0.2 & 0.3 \\
-0.1 & 0.2
\end{array}\right], \\
E_{4}=\left[\begin{array}{cc}
0.2 & 0 \\
-0.15 & 0.1
\end{array}\right], \quad d_{1}=1.2, \quad d_{2}=1.5, \quad \tau_{1}=0.3, \quad \tau_{2}=0.4, \quad \mu_{1}=0.6, \quad \mu_{2}=0.8, \quad \varepsilon=0.01
\end{gathered}
$$

According to the theorem, form (12a), (12b) by LMI toolbox in Matlab, lead to

$$
\begin{gathered}
p=\left[\begin{array}{cc}
-0.3494 & 3.7287 \\
3.7287 & -4.6081
\end{array}\right], \quad Q_{1}=10^{8}\left[\begin{array}{cc}
3.0945 & -0.0510 \\
-0.0510 & -3.1127
\end{array}\right], \quad Q_{2}=10^{8}\left[\begin{array}{cc}
4.7370 & 0.0538 \\
0.0538 & 4.7511
\end{array}\right], \\
S_{1}=10^{-3}\left[\begin{array}{cc}
0.2771 & -0.3533 \\
-0.3533 & 0.6516
\end{array}\right], \quad S_{2}=\left[\begin{array}{cc}
0.0010 & -0.0016 \\
-0.0016 & -0.0031
\end{array}\right], \quad R_{1}=10^{-3}\left[\begin{array}{cc}
0.0558 & -0.1204 \\
-0.1204 & 0.3384
\end{array}\right], \\
R_{2}=10^{-3}\left[\begin{array}{cc}
0.0446 & -0.0963 \\
-0.0963 & 0.2707
\end{array}\right], \quad N_{10}=10^{-3}\left[\begin{array}{cc}
-0.0575 & 0.2862 \\
-0.0725 & -0.0215
\end{array}\right], \quad N_{11}=\left[\begin{array}{cc}
0.0018 & 0.0010 \\
0.0006 & 0.0006
\end{array}\right], \\
N_{12}=\left[\begin{array}{cc}
-0.0001 & 0.0001 \\
-0.0013 & -0.0007
\end{array}\right], \quad M_{11}=10^{-8}\left[\begin{array}{cc}
-0.1552 & -0.1066 \\
0.2627 & 0.1663
\end{array}\right], \quad M_{12}=10^{-8}\left[\begin{array}{cc}
0.2665 & 0.2039 \\
-0.2238 & -0.1461
\end{array}\right], \\
N_{20}=10^{-3}\left[\begin{array}{cc}
-0.3006 & -0.0287 \\
0.0327 & -0.0163
\end{array}\right], \quad N_{21}=\left[\begin{array}{cc}
-0.0011 & -0.0004 \\
-0.0005 & -0.0002
\end{array}\right], \quad N_{22}=10^{-3}\left[\begin{array}{cc}
0.2400 & -0.0615 \\
0.5240 & 0.2985
\end{array}\right], \\
M_{21}=10^{-9}\left[\begin{array}{cc}
0.7342 & 0.4191 \\
-0.9933 & -0.6986
\end{array}\right], \quad M_{22}=10^{-8}\left[\begin{array}{cc}
-0.2313 & -0.1270 \\
0.0793 & 0.0460
\end{array}\right]
\end{gathered}
$$




\section{Conclusion}

The stability of neutral type descriptor systems with time-varying delays has been solved in terms of LMI approach. Using Lyapunov-Krasovskii functional method, and free-weight matrix method, a criterion for stability of systems is given. In the criterion, the relationship between $E x(t)$ and $E x\left(t-d_{i}(t)\right)-\int_{t-d_{i}(t)}^{t} E \dot{x}(s) d s$ is taken into account. The criterion is presented in terms o linear matrix inequalities, which can be easily solved by Matlab Toolbox. Finally, a numerical example is presented to illustrate the effectiveness of the method.

\section{Acknowledgements}

This article was supported by the National Science Council, Republic of China, under Grant No. 60974004. The Project was supported by the National Science Foundation of China (No. 60974004).

\section{Authors' contributions}

YZ carried out the main part of this manuscript. YM participated discussion and corrected the main theorem. All authors read and approved the final manuscript.

\section{Competing interests}

The authors declare that they have no competing interests.

Received: 3 October 2011 Accepted: 16 February 2012 Published: 16 February 2012

\section{References}

1. Park, J, Won, S: Stability analysis for neutral delay-differential systems. J Frankl Inst. 337(1):1-9 (2000). doi:10.1016/S00160032(99)00040-X

2. Chen, J, Lien, CH, Fan, KK, Chou, JH: Criteria for asymptotic stability of a class of neutral systems via a LMI approach. IEEE Proc Control Theory Appl. 148, 442-447 (2001). doi:10.1049/ip-cta:20010772

3. Li, H, Zhong, S, Li, H: Some new simple stability criteria of linear neutral systems with a single delay. J Comput Appl Math. 200(1):441-447 (2007). doi:10.1016/j.cam.2006.01.016

4. Chen, D, Jin, C: Delay-dependent stability criteria for a class of uncertain neutral systems. Acta Automatica Sinica. 34(8):989-992 (2008)

5. Han, QL: On robust stability of neutral systems with time-varying discrete delay and norm-bounded uncertainty Automatica. 40(6):1087-1092 (2004). doi:10.1016/j.automatica.2004.01.007

6. Zhao, Z, Wang, W, Yang, B: Delay and its time-derivative dependent robust stability of neutral control system. Appl Math Comput. 187(2):1326-1332 (2007). doi:10.1016/j.amc.2006.09.042

7. He, Y, Wu, M, She, JH, Liu, GP: Delay-dependent robust stability criteria for uncertain neutral systems with mixed delays. Syst Control Lett. 51(1):57-65 (2004). doi:10.1016/S0167-6911(03)00207-X

8. Li, H, Li, H, Zhong, S: Stability of neutral type descriptor system with mixted delays. Chaos Solitons Fractals. 33 1796-1800 (2007). doi:10.1016/j.chaos.2006.03.055

9. Gu, K: A further refinement of discretized Lyapunov functional method for the stability of time-delay systems. Int J Control. 7, 967-976 (2001)

10. Xu, SY, Dooren, PV, Stefan, R, Lam, J: Robust stability and stabilization for singular systems with state delay and parameter uncertainty. IEEE Trans Automat Control. 7, 1122-1128 (2002)

doi:10.1186/1687-1847-2012-15

Cite this article as: Zhao and Ma: Stability of neutral-type descriptor systems with multiple time-varying delays. Advances in Difference Equations 2012 2012:15.

\section{Submit your manuscript to a SpringerOpen ${ }^{\odot}$ journal and benefit from:}

- Convenient online submission

- Rigorous peer review

- Immediate publication on acceptance

- Open access: articles freely available online

- High visibility within the field

- Retaining the copyright to your article

Submit your next manuscript at $>$ springeropen.com 